ThE AstrophysiCal Journal, 331:350-358, 1988 August 1

(C) 1988. The American Astronomical Society. All rights reserved. Printed in U.S.A.

\title{
A LINEAR STABILITY ANALYSIS FOR WIND-DRIVEN BUBBLES
}

\author{
DONGSU RYu AND EThan T. Vishniac ${ }^{1}$ \\ Department of Astronomy, University of Texas \\ Received 1987 November 4; accepted 1988 January 25
}

\begin{abstract}
We examine the growth of linear perturbations of adiabatic shells of the shocked interstellar gas surrounding a strong stellar wind. The shells are overstable if the adiabatic index $\gamma \lesssim 1.1$, and most overstable for the wavelengths comparable to the thickness of the shell. The shells of the wind-driven bubbles are generally more stable than those of blast waves. We discuss the implications of these results.

Subject headings: hydrodynamics — instabilities — interstellar: matter — shock waves
\end{abstract}

\section{INTRODUCTION}

Stars with spectral type earlier than $B_{2}$ have strong stellar winds and blow large bubbles (Castor, McCray, and Weaver 1975; Weaver et al. 1977). Initially, the stellar wind expands freely. After the wind sweeps up an amount of the ambient interstellar gas roughly equal in mass to its own ejecta, the system quickly evolves into a four-zone structure. Starting from the innermost region, the four zones are $(a)$ a region of hypersonic stellar wind, $(b)$ a region of shocked stellar wind, $(c)$ a shell of shocked interstellar wind, and $(d)$ the ambient interstellar gas. The evolutionary history of the bubble may be divided into three stages. The first stage is a fully adiabatic stage in which the bubble expands so fast that the radiative losses in the gas interior to the bubble have negligible effects on the dynamics of the system. In the second stage, the shocked interstellar gas cools and forms a thin and dense shell, but the shocked stellar wind is still so hot that the radiative cooling is not important. In the final stage, the radiative cooling in the shocked stellar wind becomes important and affects the dynamics of the system.

In our paper, we will discuss the dynamic instability for the shells of the shocked interstellar gas in the fully adiabatic stage for several values of adiabatic index, $\gamma$, and compare the results with those for the shell of blast waves (Ryu and Vishniac 1987). The main difference between wind-driven bubbles and blast waves is that the radius $r_{2}$ of the shock front changes as

$$
r_{2} \propto t^{3 / 5}
$$

for wind-driven bubbles and as

$$
r_{2} \propto t^{2 / 5}
$$

for blast waves. Here, we will consider a somewhat idealized case without conductivity, viscosity, and magnetic field. Even though the adiabatic shell of the shocked interstellar gas has $\gamma=5 / 3$, if the radiative cooling of the shell becomes important, the thickness of the shell and the effective value of $\gamma$ reduce. In $\S$ II we present the conservation equations governing the gasflow motion of the shocked interstellar gas and the solutions for the unperturbed structure of the adiabatic shells. In $\S$ III we calculate the frequency spectra of an infinitely thin shell which corresponds to $\gamma=1$, in order to compare them with those for a shell with finite thickness. In $\S$ IV we derive the perturbation equations and the boundary conditions for the perturbed shell

\footnotetext{
${ }^{1}$ Presidential Young Investigator and Alfred P. Sloan Fellow.
}

of the shocked interstellar gas and solve them to find the frequency spectra for several values of $\gamma$. Finally, in $\S \mathrm{V}$ we discuss the implications of this work.

\section{UNPERTURBED SHELL OF THE SHOCKED INTERSTELLAR GAS}

The structure of the shocked interstellar gas in the fully adiabatic stage is described by a similarity solution, which is analogous to that of the adiabatic blast wave for a point explosion (Sedov 1959). If the propagation speed of the outer shock front greatly exceeds the sound speed of the ambient interstellar gas, the boundary conditions just behind the shock front can be derived from the strong-shock jump conditions:

$$
\begin{aligned}
& \rho_{2}=\frac{\gamma+1}{\gamma-1} \rho_{1}, \\
& v_{2}=\frac{2}{\gamma+1} u_{1}, \\
& p_{2}=\frac{2}{\gamma+1} \rho_{1} u_{1}^{2},
\end{aligned}
$$

where $\rho_{1}$ is the density of the ambient gas and $u_{1}$ is the velocity of the shock front. The similarity parameter for the winddriven bubble which is formed from the mechanical energy $L_{w}$ of the wind, the density $\rho_{1}$, and the two independent variables $r$ and $t$ is

$$
\xi=r\left(\frac{\rho_{1}}{L_{w} t^{3}}\right)^{1 / 5} .
$$

If we introduce the normalized variables defined by

$$
\begin{aligned}
\tilde{\rho} & \equiv \frac{\rho}{\rho_{2}}, \\
\tilde{v} & \equiv \frac{v}{v_{2}}, \\
\tilde{p} & \equiv \frac{p}{p_{2}}, \\
\tilde{r} & \equiv \frac{\xi}{\xi_{2}} \equiv \frac{r}{r_{2}},
\end{aligned}
$$

where $\xi_{2}$ is the value of the similarity parameter at the shock front and $r_{2}$ is the radius of the shock front, the hydrodynamic 
equations become

$$
\begin{gathered}
\left(\frac{2 \tilde{v}}{\gamma+1}-\tilde{r}\right) \frac{1}{\tilde{\rho}} \frac{d \tilde{\rho}}{d \tilde{r}}+\frac{2}{\gamma+1} \frac{d \tilde{v}}{d \tilde{r}}+\frac{4}{\gamma+1} \frac{\tilde{v}}{\tilde{r}}=0, \\
\left(\frac{2 \tilde{v}}{\gamma+1}-\tilde{r}\right) \frac{d \tilde{v}}{d \tilde{r}}+\frac{\gamma-1}{\gamma+1} \frac{1}{\tilde{\rho}} \frac{d \tilde{p}}{d \tilde{r}}-\frac{2}{3} \tilde{v}=0, \\
-\gamma\left(\frac{2 \tilde{v}}{\gamma+1}-\tilde{r}\right) \frac{1}{\tilde{\rho}} \frac{d \tilde{\rho}}{d \tilde{r}}+\left(\frac{2 \tilde{v}}{\gamma+1}-\tilde{r}\right) \frac{1}{\tilde{p}} \frac{d \tilde{p}}{d \tilde{r}}-\frac{4}{3}=0 .
\end{gathered}
$$

Here, we used the relations

$$
\begin{aligned}
& \frac{\partial \tilde{\rho}}{\partial t}=-\frac{3}{5} \frac{\tilde{r}}{t} \frac{d \tilde{\rho}}{d \tilde{r}}, \\
& \frac{\partial \tilde{v}}{\partial t}=-\frac{3}{5} \frac{\tilde{r}}{t} \frac{d \tilde{v}}{d \tilde{r}}, \\
& \frac{\partial \tilde{p}}{\partial t}=-\frac{3}{5} \frac{\tilde{r}}{t} \frac{d \tilde{p}}{d \tilde{r}} .
\end{aligned}
$$

The above equations with the boundary conditions at the shock front $\tilde{\rho}=\tilde{v}=\tilde{p}=1$ were integrated, using a fifth- and sixth-order Runge-Kutta method with 5000 points between the shock front and the contact discontinuity. We used $\ln \tilde{\rho}$ intead of $\tilde{r}$ as the independent parameter, in order to improve our numerical accuracy around the contact discontinuity. The results are shown in Table 1 and Figure 1 . As expected, the thickness of the shell between the shock front and the contact discontinuity decreases with $\gamma$. Around the contact discontinuity, the density plummets sharply to zero, while the pressure approaches a constant value. The shell structure for the shocked interstellar gas of the wind-driven bubble is quite different from that for the blast wave.

\section{STABILITY OF AN INFINITELY THIN SHELL}

Table 1 shows that the shell of the shocked interstellar gas becomes infinitely thin as $\gamma$ approaches 1 . In this limit, the effect of the internal gas pressure of the shell can be neglected and the stability spectrum can be computed from the continuity equation and the momentum equation. Following Vishniac (1983) and Ryu and Vishniac (1987), we will consider three variables: the surface density,

\begin{tabular}{|c|c|}
\hline$\gamma$ & $\tilde{r}_{c}^{a}$ \\
\hline 1.05 & 0.983 \\
\hline 1.1. & 0.968 \\
\hline 1.2 & 0.942 \\
\hline …… & 0.920 \\
\hline 1.4 & 0.900 \\
\hline 1.5 & 0.883 \\
\hline 1.667 . & 0.858 \\
\hline
\end{tabular}

$$
\sigma \equiv \frac{1}{r_{s}^{2}} \int_{r=0}^{r=r_{s}} \rho r^{2} d r
$$

\section{TABLE 1}

Position OF THE CONTACT DisCONTINUITY

${ }^{a}$ The relative position of the contact discontinuity with respect to the radius of the shock front. where $r_{s}$ is the radius of the shell; the radial velocity of the gas, $v_{r}$; and the tangential velocity of the gas, $v_{T}$. We define the perturbation variables by

$$
\begin{gathered}
\delta \equiv \frac{\sigma-\sigma_{0}}{\sigma_{0}}, \\
\Delta r \equiv r_{s}-r_{2},
\end{gathered}
$$

where $\sigma_{0}$ is the average surface density and $r_{2}$ is the average radius of the shell. If we expand the spatial dependence of the perturbation variables in spherical harmonics and the time dependence in a power law,

$$
\begin{aligned}
\delta & \propto Y_{l m}(\theta, \phi) t^{s}, \\
\frac{\Delta r}{r_{2}} & \propto Y_{l m}(\theta, \phi) t^{s}, \\
\frac{v_{T}}{v_{0}} & \propto Y_{l m}(\theta, \phi) t^{s},
\end{aligned}
$$

where $v_{0}$ is the average radial velocity of the shell, then the continuity equation and the momentum equation give

$$
(s+2)\left(s+\frac{9}{5}\right)\left(s^{2}+\frac{19}{5} s+\frac{42}{25}\right)+\frac{126}{625} l(l+1)=0 .
$$

Here, we used the relation

$$
r_{2} \propto t^{3 / 5} .
$$

Figures $2 a$ and $2 b$ show the real and imaginary parts of the growing mode of $s$ as functions of $l$, and Figures $2 c$ and $2 d$ show those of the decaying mode of $s$. From Figure $2 a$, the shell of the wind-driven bubble is overstable for $l \gtrsim-\left(\frac{1}{2}\right)$ $+\left([2327]^{1 / 2} / 2[3]^{1 / 2}\right)$ with the oscillatorily growing perturbation. On the other hand, the shell of the blast wave is overstable for $l \gtrsim-\left(\frac{1}{2}\right)+\left([877]^{1 / 2} / 2[3]^{1 / 2}\right)$ (Ryu and Vishniac 1987).

\section{STABILITY OF A SHELL WITH FINITE THICKNESS}

\section{a) Perturbation Equations}

Following Ryu and Vishniac (1987), let us consider the normalized perturbation variables defined by

$$
\begin{aligned}
\delta \tilde{\rho}(r, \theta, \phi, t) & =\frac{\rho(r, \theta, \phi, t)-\rho_{0}(r, t)}{\rho_{2}}, \\
\delta \tilde{v}(r, \theta, \phi, t) & \equiv \frac{v(r, \theta, \phi, t)-v_{0}(r, t) \hat{r}}{v_{2}} \\
\delta \tilde{p}(r, \theta, \phi, t) & \equiv \frac{p(r, \theta, \phi, t)-p_{0}(r, t)}{p_{2}}
\end{aligned}
$$

where $\rho_{0}, v_{0}$, and $p_{0}$ are the unperturbed quantities considered in $\S$ II. Since the unperturbed shell of the shocked interstellar gas is a similarity flow, the normalized unperturbed variables are functions of only a single normalized similarity parameter, $\tilde{r}$,

$$
\begin{aligned}
& \tilde{\rho}=\tilde{\rho}(\tilde{r}), \\
& \tilde{v}=\tilde{v}(\tilde{r}), \\
& \tilde{p}=\tilde{p}(\tilde{r}),
\end{aligned}
$$

and we can assume that the normalized perturbation variables are composed of the similarity term which is a function of a 

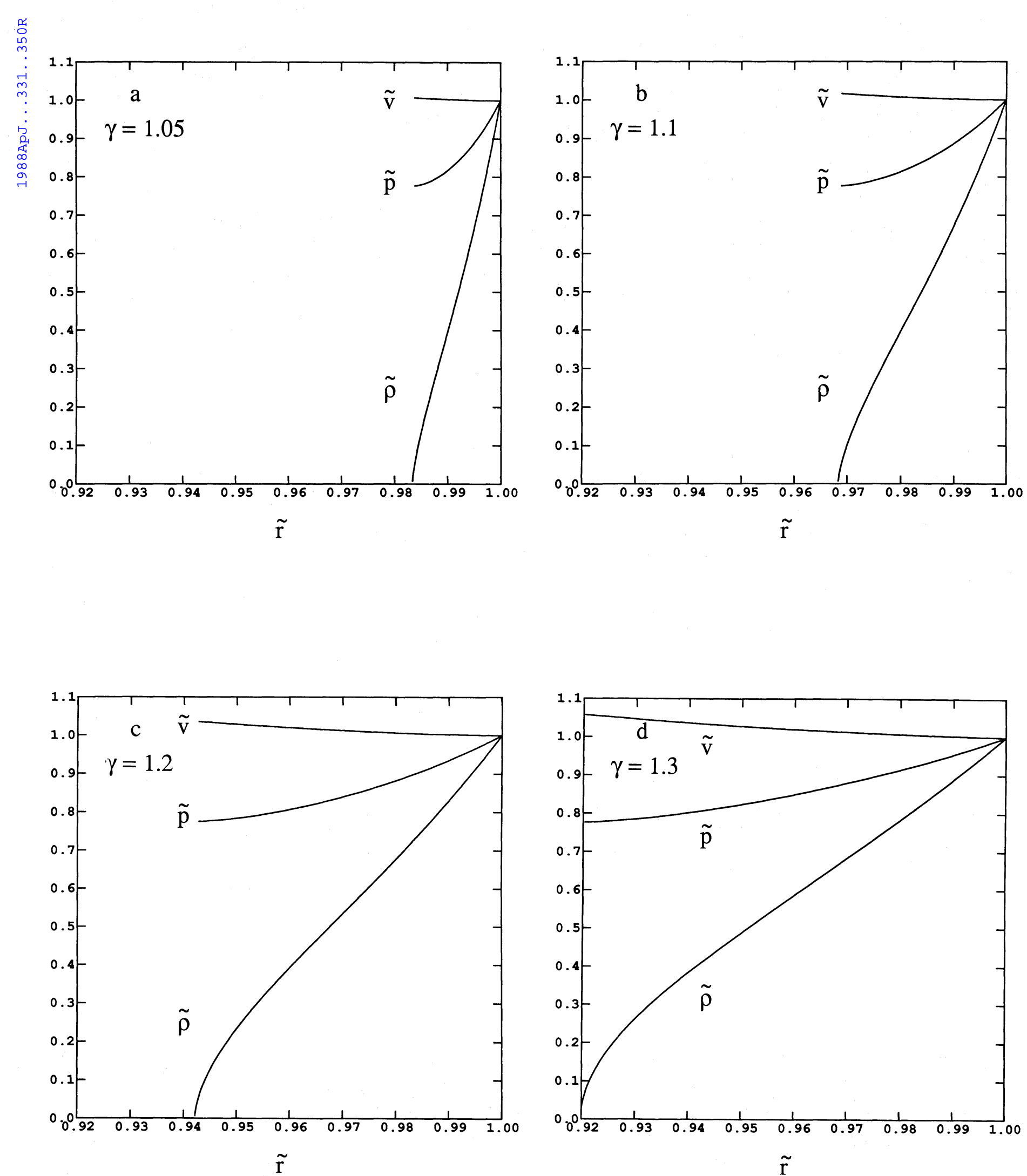

Fig. 1.-The distributions of the normalized quantities $\tilde{\rho}, \tilde{v}, \tilde{p}$ as functions of the normalized similarity parameter $\tilde{r}$ for the shell of the shocked interstellar gas in the fully adiabatic stage; $(a),(b),(c)$, and $(d)$ correspond to $\gamma=1.05,1.1,1.2$, and 1.3 , respectively. 


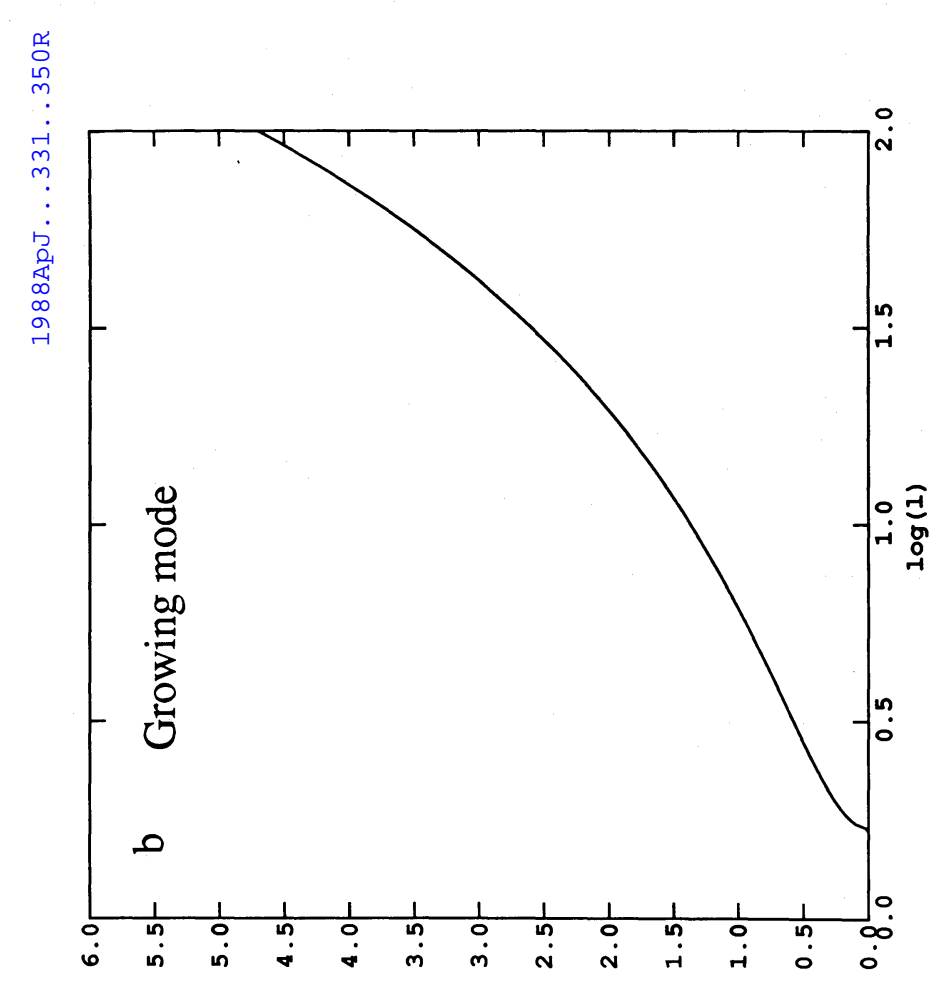

(s) beur?

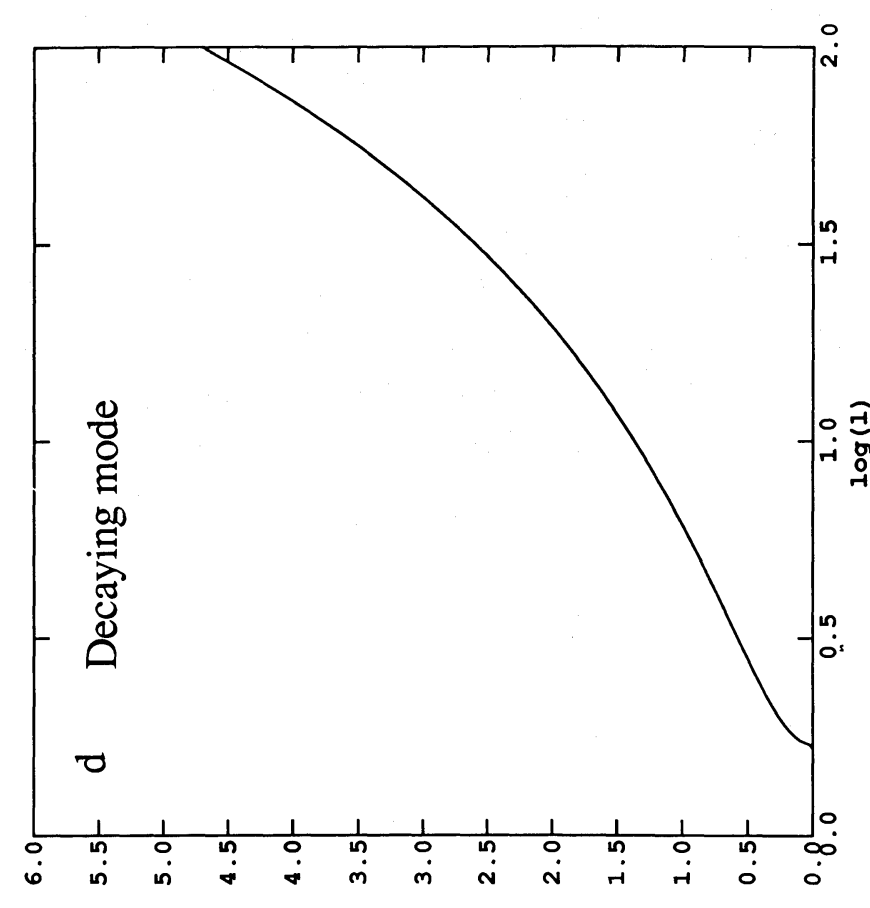

(s) beur?

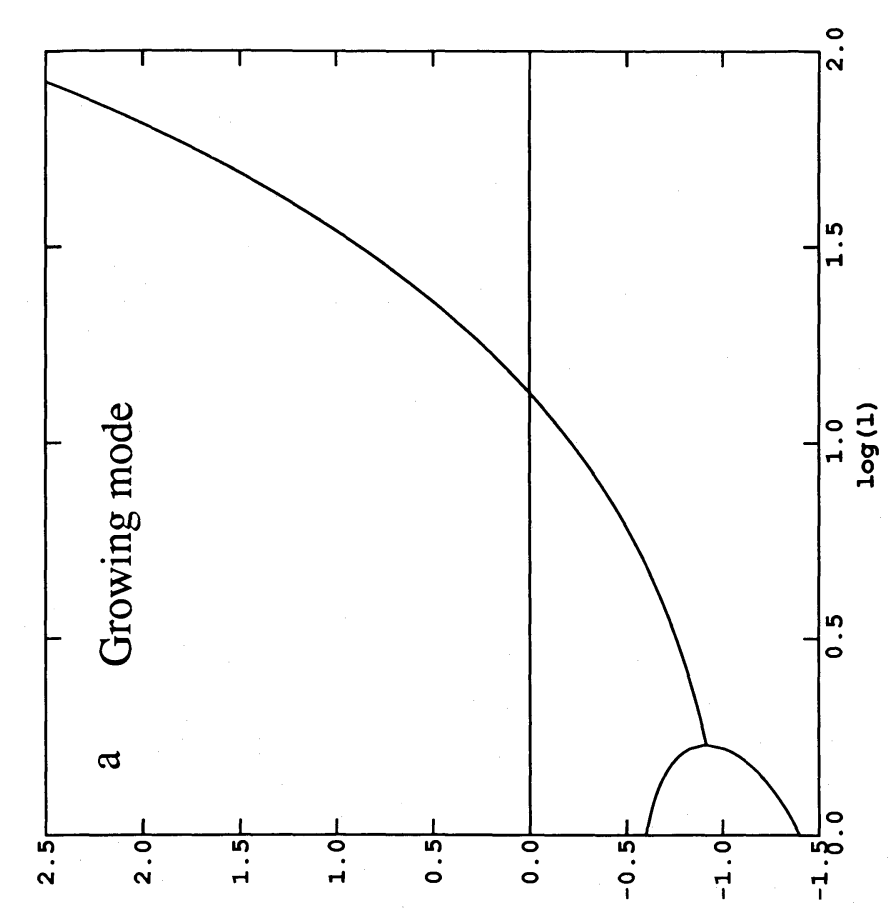

(s) teax

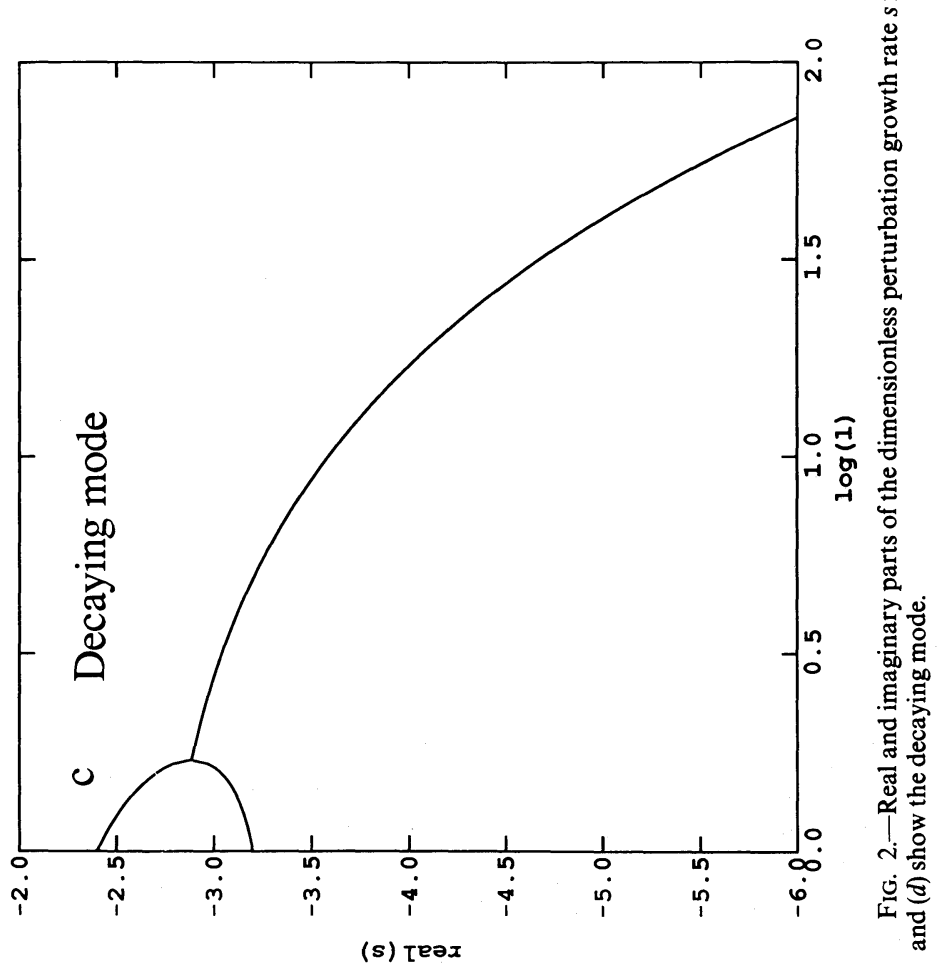

353 
normalized similarity parameter, the spatial dependence in spherical harmonics, and the time dependence in power law,

$$
\begin{aligned}
\delta \tilde{\rho} & =\delta \tilde{\rho}(\tilde{r}) Y_{l m}(\theta, \phi) t^{s}, \\
\delta \tilde{v} & =\delta \tilde{v}_{r}(\tilde{r}) Y_{l m}(\theta, \phi) t^{s} \hat{r}+\delta \tilde{v}_{T}(\tilde{r}) \tilde{r} \tilde{\nabla}_{T} Y_{l m}(\theta, \phi) t^{s}, \\
\delta \tilde{p} & =\delta \tilde{p}(\tilde{r}) Y_{l m}(\theta, \phi) t^{s},
\end{aligned}
$$

where the operator $\tilde{\nabla}_{T}$ is defined by

$$
\tilde{\nabla}_{T} \equiv \frac{1}{\tilde{r}} \frac{\partial}{\partial \theta} \hat{\boldsymbol{\theta}}+\frac{1}{\tilde{r} \sin \theta} \frac{\partial}{\partial \phi} \hat{\boldsymbol{\phi}}
$$

Then, by linearizing the hydrodynamic equations about the perturbation variables, we obtain the perturbation equations for the shocked interstellar gas region:

$$
\begin{aligned}
& \left(-\frac{\gamma+1}{2} \tilde{r}+\tilde{v}\right) \frac{d \delta \tilde{\rho}}{d \tilde{r}}+\tilde{\rho} \frac{d \delta \tilde{v}_{r}}{d \tilde{r}}+\left[\frac{5(\gamma+1)}{6} s+2 \frac{\tilde{v}}{\tilde{r}}+\frac{d \tilde{v}}{d \tilde{r}}\right] \delta \tilde{\rho} \\
& +\left(2 \frac{\tilde{\rho}}{\tilde{r}}+\frac{d \tilde{\rho}}{d \tilde{r}}\right) \delta \tilde{v}_{r}-l(l+1) \frac{\tilde{\rho}}{\tilde{r}} \delta \tilde{v}_{T}=0, \\
& \left(-\frac{\gamma+1}{2} \tilde{r}+\tilde{v}\right) \tilde{\rho} \frac{d \delta \tilde{v}_{r}}{d \tilde{r}}+\frac{\gamma-1}{2} \frac{d \delta \tilde{p}}{d \tilde{r}}-\frac{\gamma-1}{2} \frac{1}{\tilde{\rho}} \frac{d \tilde{p}}{d \tilde{r}} \delta \tilde{p} \\
& +\left[\frac{5(\gamma+1)}{6}\left(s-\frac{2}{5}\right)+\frac{d \tilde{v}}{d \tilde{r}}\right] \tilde{\rho} \delta \tilde{v}_{r}=0, \\
& \left(-\frac{\gamma+1}{2} \tilde{r}+\tilde{v}\right) \tilde{\rho} \frac{d \delta \tilde{v}_{T}}{d \tilde{r}}+\frac{\gamma-1}{2} \frac{1}{\tilde{r}} \delta \tilde{p} \\
& +\left[\frac{5(\gamma+1)}{6}\left(s-\frac{2}{5}\right)+\frac{\tilde{v}}{\tilde{r}}\right] \tilde{\rho} \delta \tilde{v}_{T}=0 \\
& -\gamma\left(-\frac{\gamma+1}{2} \tilde{r}+\tilde{v}\right) \frac{d \delta \tilde{\rho}}{d \tilde{r}}+\frac{\tilde{\rho}}{\tilde{p}}\left(-\frac{\gamma+1}{2} \tilde{r}+\tilde{v}\right) \frac{d \delta \tilde{p}}{d \tilde{r}} \\
& +\left(-\gamma \frac{1}{\tilde{\rho}} \frac{d \tilde{\rho}}{d \tilde{r}}+\frac{1}{\tilde{p}} \frac{d \tilde{p}}{d \tilde{r}}\right) \tilde{\rho} \delta \tilde{v}_{r} \\
& -\gamma\left[\frac{5(\gamma+1)}{6} s-\left(-\frac{\gamma+1}{2} \tilde{r}+\tilde{v}\right) \frac{1}{\tilde{\rho}} \frac{d \tilde{\rho}}{d \tilde{r}}\right] \delta \tilde{\rho} \\
& +\frac{\tilde{\rho}}{\tilde{p}}\left[\frac{5(\gamma+1)}{6} s-\left(-\frac{\gamma+1}{2} \tilde{r}+\tilde{v}\right) \frac{1}{\tilde{p}} \frac{d \tilde{p}}{d \tilde{r}}\right] \delta \tilde{p}=0
\end{aligned}
$$

Here, we used the relations $(2.5 \mathrm{a})-(2.5 \mathrm{c})$ and

$$
\begin{aligned}
& \frac{\partial \delta \tilde{\rho}}{\partial t}=-\frac{3}{5} \frac{\tilde{r}}{t} \frac{\partial \delta \tilde{\rho}}{\partial \tilde{r}}+\frac{s}{t} \delta \tilde{\rho}, \\
& \frac{\partial \delta \tilde{v}}{\partial t}=-\frac{3}{5} \frac{\tilde{r}}{t} \frac{\partial \delta \tilde{v}}{\partial \tilde{r}}+\frac{s}{t} \delta \tilde{v}, \\
& \frac{\partial \delta \tilde{p}}{\partial t}=-\frac{3}{5} \frac{\tilde{r}}{t} \frac{\partial \delta \tilde{p}}{\partial \tilde{r}}+\frac{s}{t} \delta \tilde{p}
\end{aligned}
$$

Although we do not distinguish notationally between, say, $\delta \tilde{\rho}(\tilde{r}, \theta, \phi, t)$ and $\delta \tilde{\rho}(\tilde{r})$, the latter form is applied in equations $(4.5 \mathrm{a})-(4.5 \mathrm{~d})$.

\section{b) Boundary Conditions}

The outer boundary conditions at the shock front for the perturbation equations $(4.5 \mathrm{a})-(4.5 \mathrm{~d})$ can be derived by the same method as in Ryu snd Vishniac (1987). From the requirement of the conservations of mass, momentum, and energy, we have

$$
\begin{aligned}
{\left[\rho u_{r}\right] } & =0, \\
{\left[p+\rho u_{r}^{2}\right] } & =0, \\
{\left[u_{T}\right] } & =0, \\
{\left[\frac{1}{2} u^{2}+\frac{\gamma}{\gamma-1} \frac{p}{\rho}\right] } & =0,
\end{aligned}
$$

where $u_{r}$ is the radial gas velocity relative to the shock front, $u_{T}$ is the gas velocity tangential to the shock front, and the square brackets denote the difference in the enclosed quantities across the shock front. If we consider the normalized perturbation of the position of the shock front

$$
\tilde{\eta} \equiv \frac{\eta}{r_{2}}
$$

and assume that it has the spatial dependence proportional to spherical harmonics and the time dependence proportional to power law

$$
\tilde{\eta}=\tilde{\eta} Y_{l m}(\theta, \phi) t^{s},
$$

the equations $(4.7 \mathrm{a})-(4.8 \mathrm{~d})$ give the normalized boundary conditions at the shock front

$$
\begin{aligned}
\delta \tilde{\rho} & =-\tilde{\eta} \frac{d \tilde{\rho}}{d \tilde{r}}, \\
\delta \tilde{v}_{r} & =-\tilde{\eta} \frac{d \tilde{v}}{d \tilde{r}}+\tilde{\eta}+\frac{5}{3} s \tilde{\eta}, \\
\delta \tilde{p} & =-\tilde{\eta} \frac{d \tilde{p}}{d \tilde{r}}+2 \tilde{\eta}+\frac{10}{3} s \tilde{\eta}, \\
\delta \tilde{v}_{T} & =-\tilde{\eta} .
\end{aligned}
$$

Here, $\tilde{\eta}$ behaves as a scale factor which we set conveniently to equal 1 .

The derivation of the inner boundary condition at the contact discontinuity is subtler than that of the outer boundary conditions, and the physical arguments for the inner boundary condition are somewhat different from those of the blast wave case (Ryu and Vishniac 1987). In the region of the shocked stellar wind, the gas pressure is finite while the gas density is very small. Hence, we can assume that the sound speed is so large that all the perturbations in the contact discontinuity immediately smooth out. Requiring pressure continuity across the contact discontinuity yields the inner boundary condition

$$
\delta \tilde{p}=0 \text {. }
$$

However, it is not clear whether the above argument is still applicable even when the perturbation wavelength $\lambda \sim 2 \pi r_{2} / l$ is comparable to, or larger than, the thickness of the region of the shocked stellar wind. Fortunately, it is known that the accretion of the interstellar gas onto the shell makes the shell stable against perturbations with wavelengths larger than the thickness of the shocked stellar wind region. Therefore, even the unphysical inner boundary condition for such wavelengths does not affect our main results.

\section{c) Numerical Calculations}

The eigenvalue equations $(4.5 \mathrm{a})-(4.5 \mathrm{~d})$ were solved by an iteration method. For given values of $\gamma$ and $l$, we first guess the 


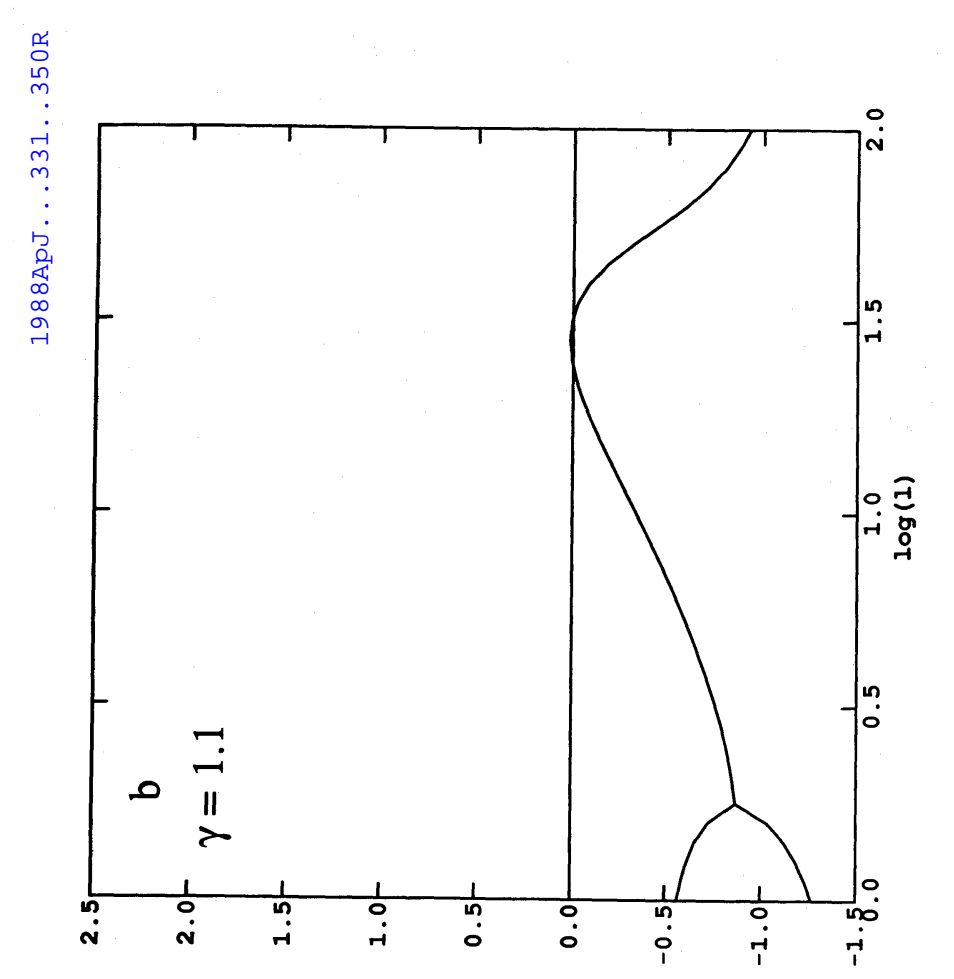

(s) โеəx

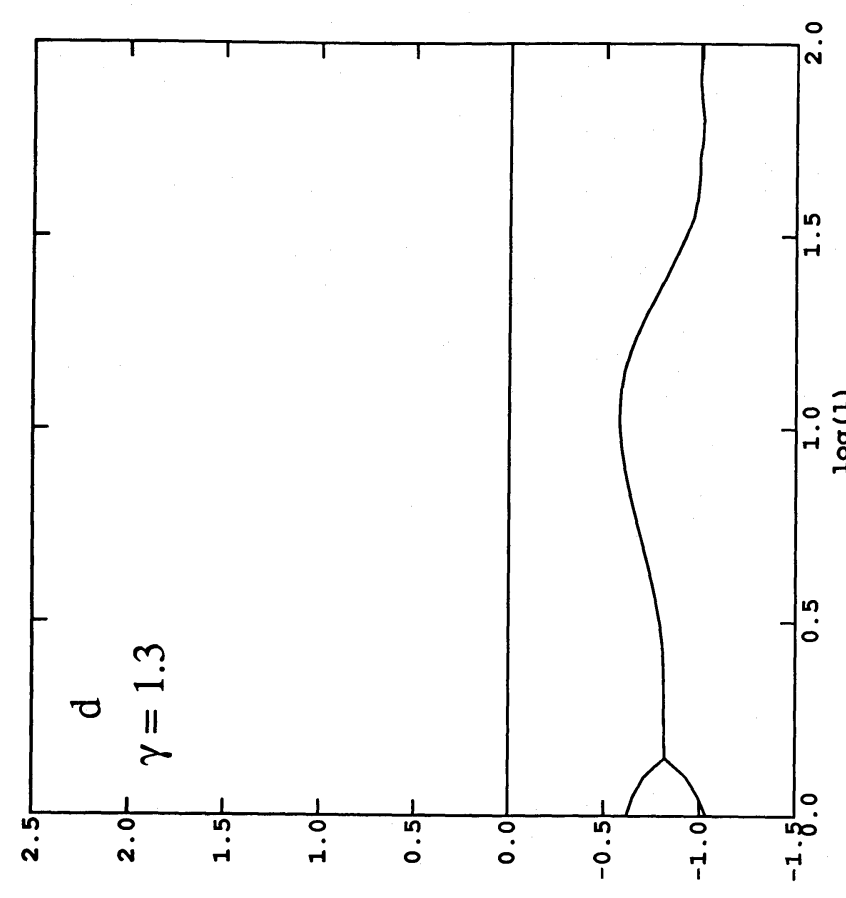

(s) โeax
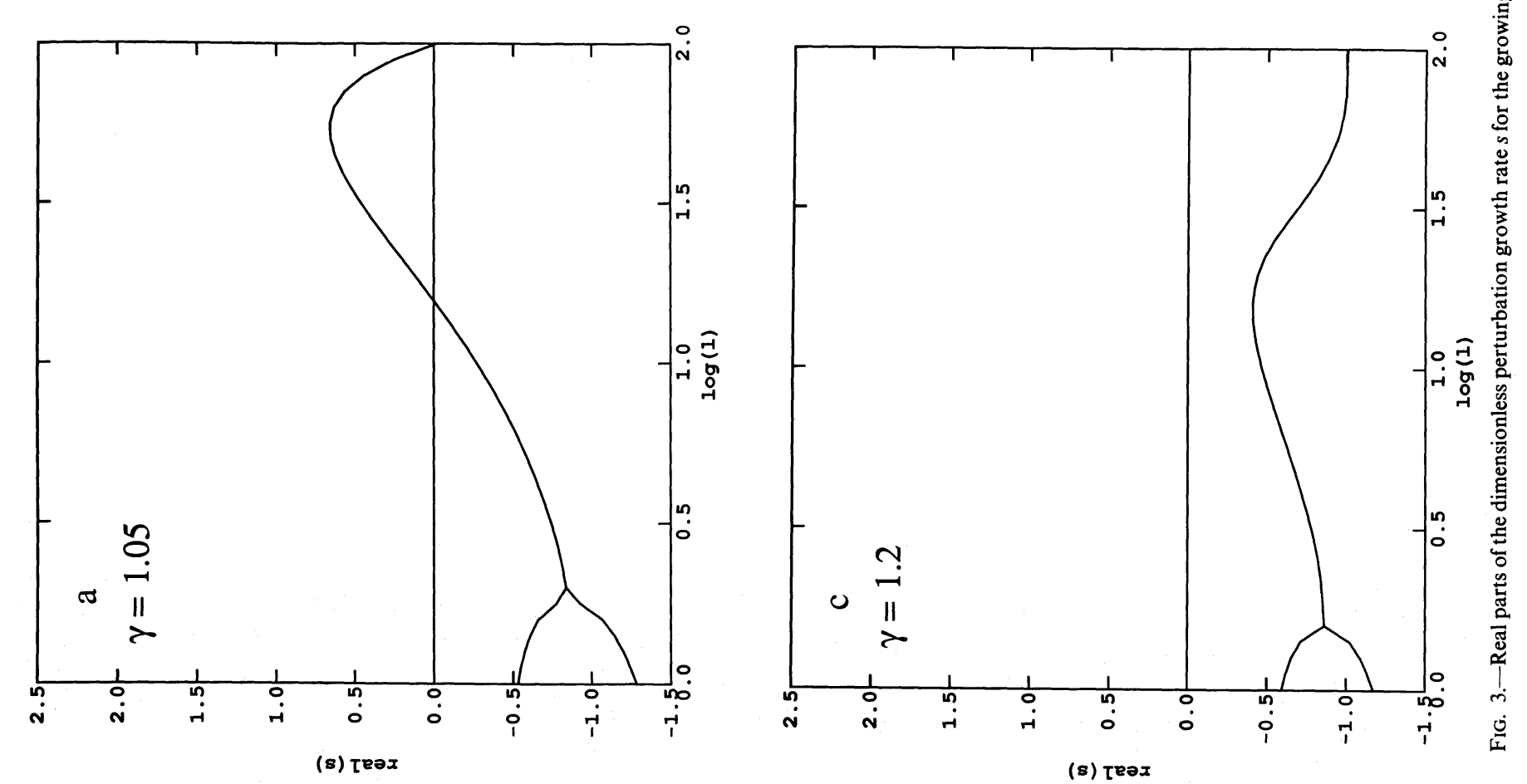

(s) teax 


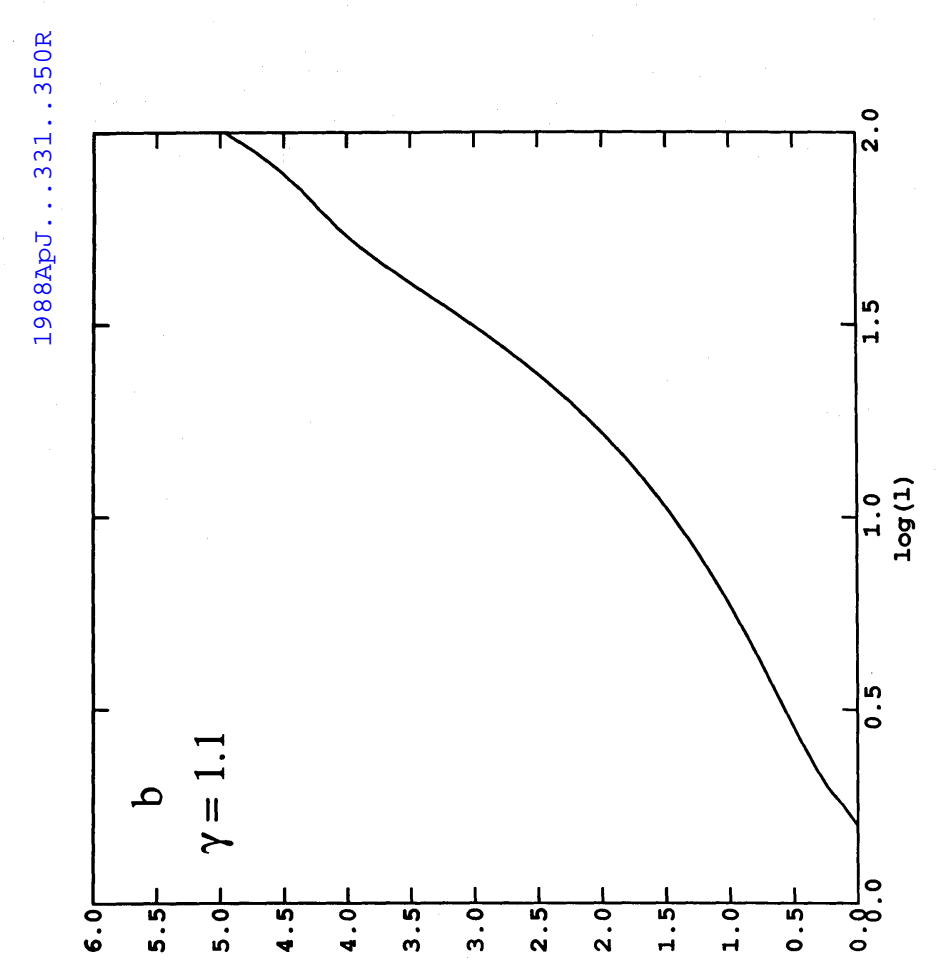

(s) bew?

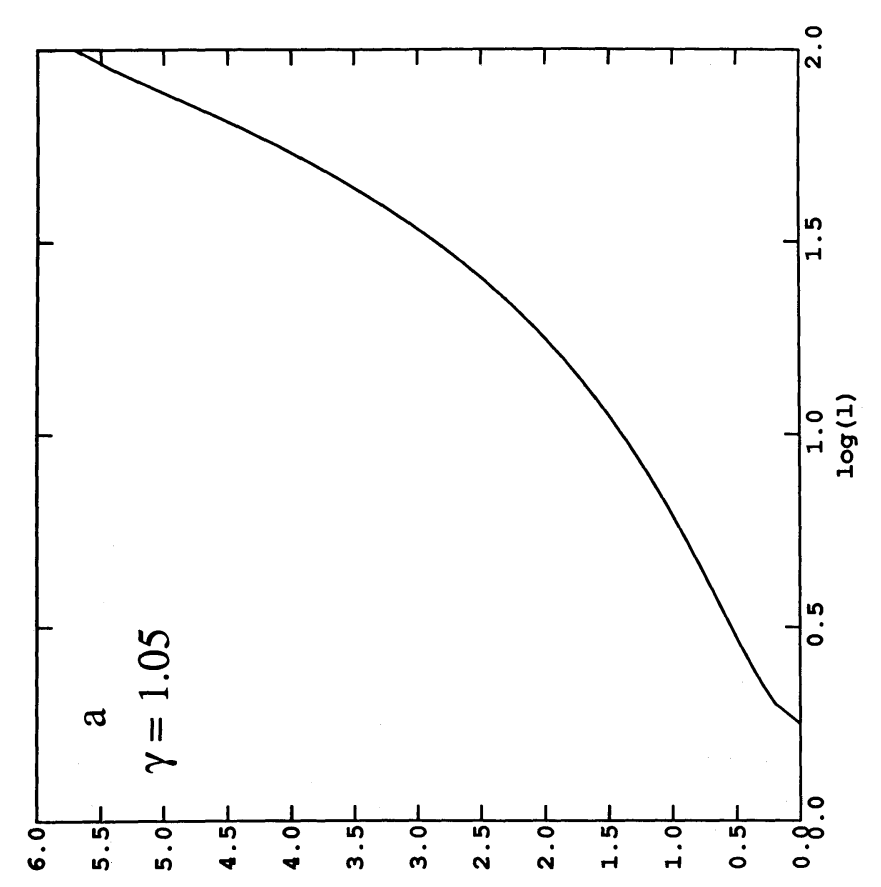

(s) Geur?

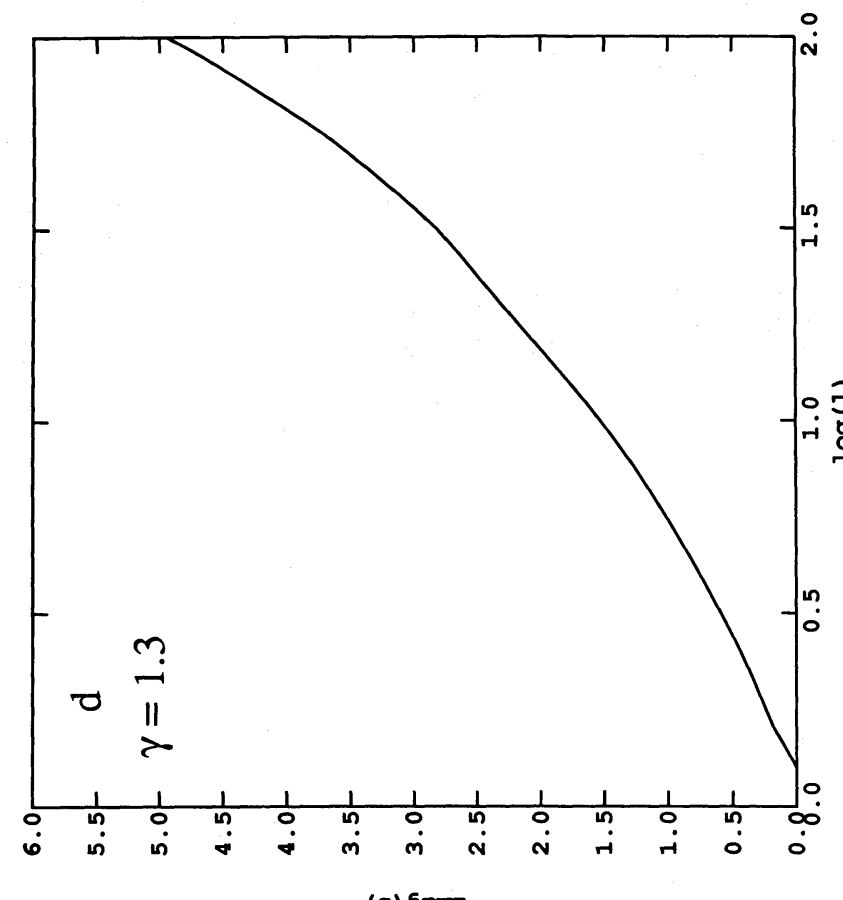

(s) 6ewt

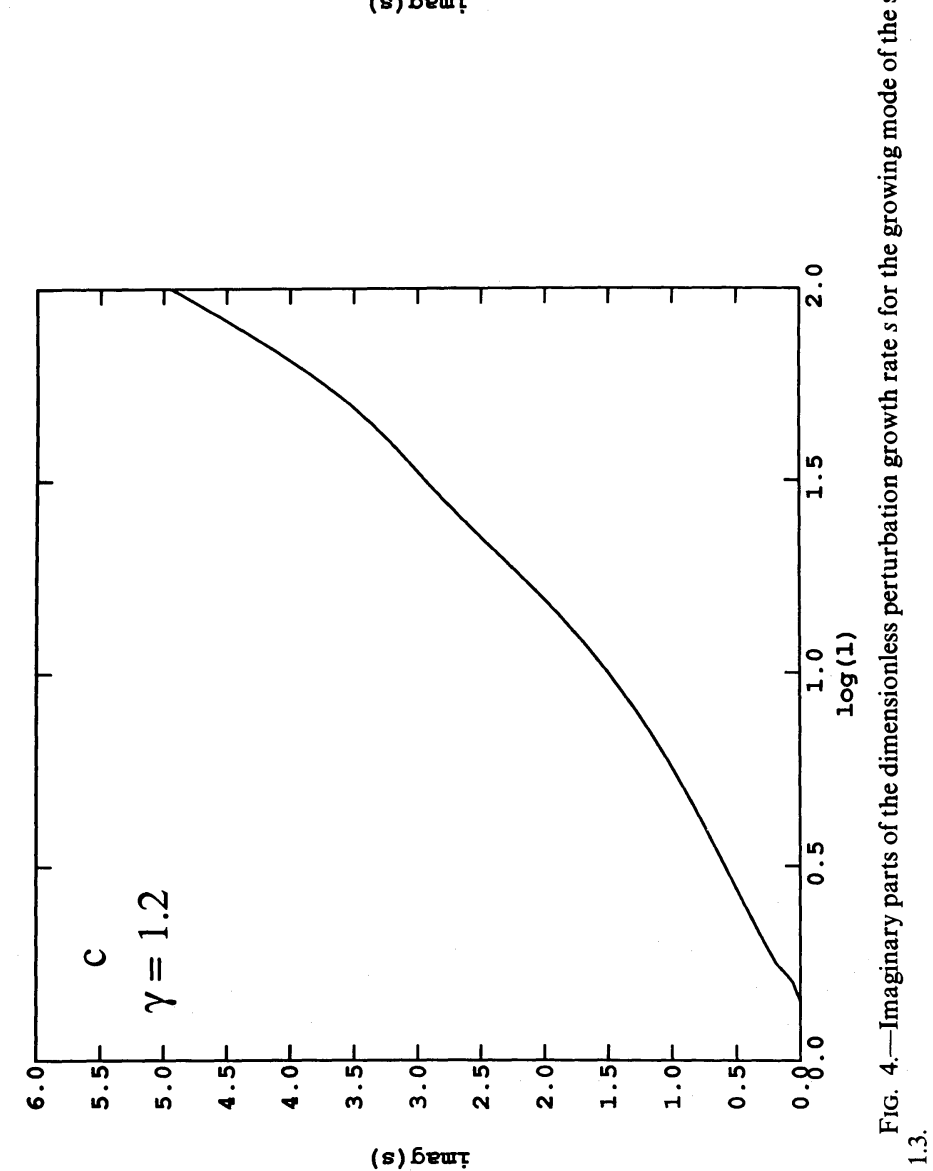


eigenvalue $s$ from the frequency spectra for the infinitely thin shell in $\S$ III. With the outer boundary conditions at the shock front (eqs. [4.10a]-[4.10d]), the equations were integrated up to the contact discontinuity using a fifth- and sixth-order Runge-Kutta method with 5000 points, and the result was checked against the inner boundary condition. If $\delta \tilde{p} \neq 0, s$ was modified to a better value $s+\Delta s$ by the formula

$$
\Delta s=-\frac{\delta \tilde{p}\left(\tilde{r}=\tilde{r}_{c}\right)}{(\partial \delta \tilde{p} / \partial s)\left(\tilde{r}=\tilde{r}_{c}\right)}
$$

and iterated until $|\Delta s|$ changed less than $10^{-4}$ per iteration. Typically, $s$ converges in three or four iterations for given values of $\gamma$ and $l$. As before, we used $\ln \tilde{\rho}$ instead of $\tilde{r}$ as the independent parameter in order to improve the accuracy around the contact discontinuity. The resulting frequency spectra of the eigenvalue $s$ which correspond to the growing mode of the infinitely thin shell of $\S$ III are shown in Figures 3 and 4.

The shell of the shocked interstellar gas in the fully adiabatic stage is overstable for $\gamma \lesssim 1.1$. The shell with $\gamma=1.1$ is marginally overstable around the wavelengths corresponding to $l \approx 28$. The shell with $\gamma=1.05$ is overstable in the range of wavelengths corresponding to $16 \lesssim l \lesssim 89$ and most overstable for a wavelength corresponding to $l=56$. On the other hand, the adiabatic shock of the blast wave is overstable for $\gamma \lesssim 1.2$, and the shock with $\gamma=1.1$ is most overstable for a wavelength corresponding to $l \approx 40$ (Ryu and Vishniac 1987). The shell behind the shock front is generally stable against perturbations with small wavelengths, because the internal pressure perturbation prevents the perturbations from growing further. However, the internal pressure perturbation becomes dynamically negligible for the wavelengths much longer than the thickness of the shell.
Figures $5 a$ and $5 b$ show the distributions of the perturbation variables $\delta \tilde{\rho}, \delta \tilde{v}_{r}, \delta \tilde{p}$, and $\delta \tilde{v}_{T}$ within the shell of the shocked interstellar gas for an oscillatorily growing mode with $\gamma=1.05$, $\log (l)=1.75$, and eigenvalue $s=0.657+4.102 i$. As expected, the pressure perturbation converges to zero around the contact discontinuity, while the density perturbation diverges to infinity. This singular behavior may look surprising, but the density perturbation is actually cancelled out at the contact discontinuity with $-\tilde{\eta}_{c} d \tilde{\rho} / d \tilde{r}$ which also diverges to infinity, where $\tilde{\eta}_{c}$ is the normalized perturbation of the position of the contact discontinuity.

\section{DISCUSSION}

In this paper, we have examined the growth of perturbations of adiabatic shells of wind-driven bubbles and shown that the shells of the shocked interstellar gas are overstable for $\gamma \lesssim 1.1$. In the real interstellar medium, of course, the shocked gas has $\gamma=5 / 3$ and the adiabatic shell will be stable. However, once the time scale for the radiative cooling of the shocked interstellar gas becomes comparable to the age of the bubble, the temperature and the thickness of the shell will decrease and the gas will act as if it had an effective $\gamma$ close to 1 . Although a radiative shell has an internal structure somewhat different from that of an adiabatic shell with $\gamma$ close to 1 , since the overstabilty mainly depends on the thickness of the shell, the radiative shell is expected to be overstable for wavelengths comparable to the thickness of the shell or larger.

When the radiative shell is unstable in the linear regime, the perturbation is expected to grow until the nonlinear effects become important. From the linear analysis which we have done so far, we know nothing about the nonlinear effects. If the shell is still unstable in the nonlinear regime, it will even-

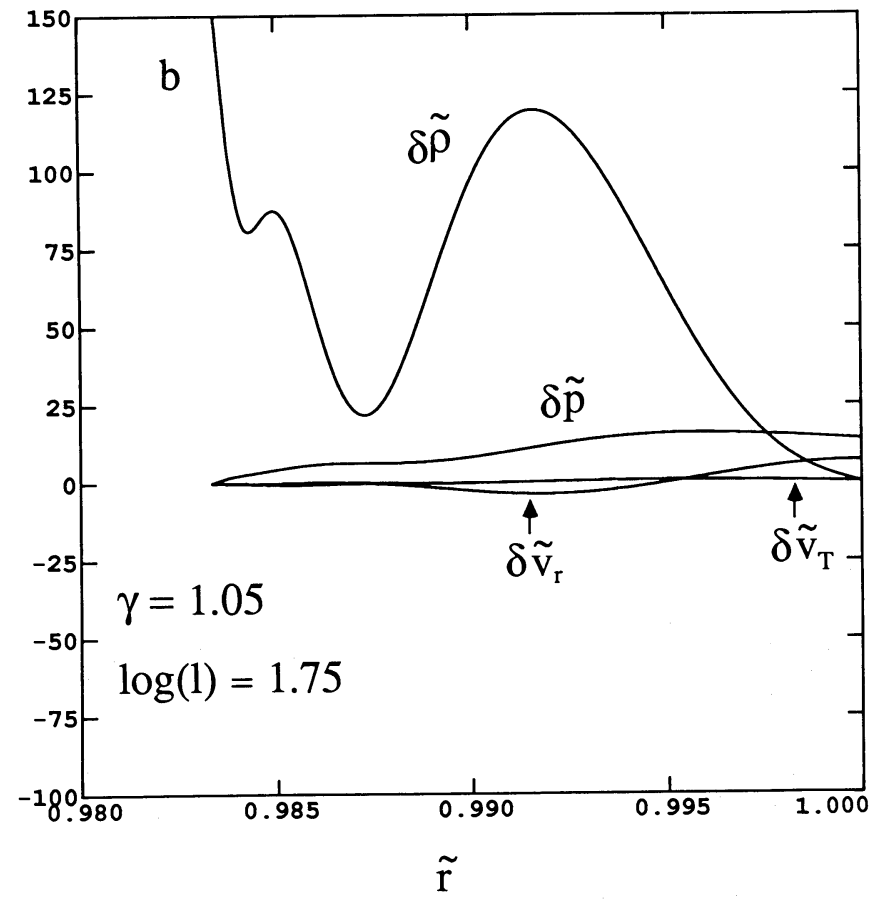

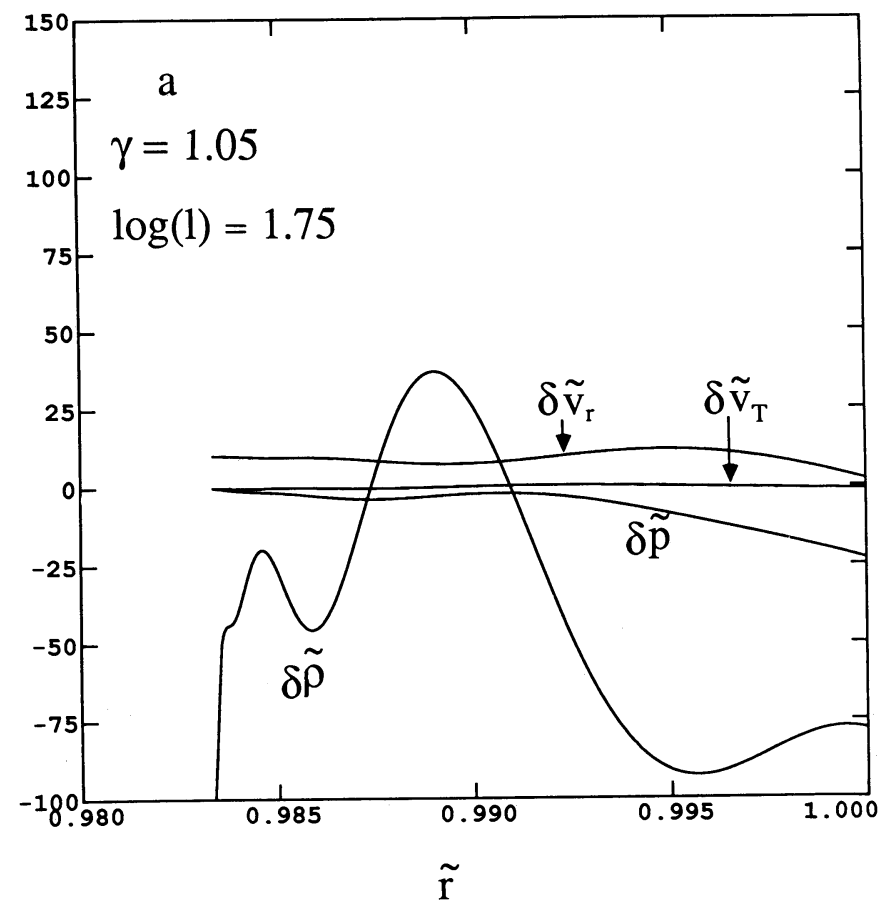

Fig. 5.- - Real (a) and imagninary $(b)$ parts of the normalized perturbation quantities $\delta \tilde{\rho}, \delta \tilde{v}_{r}, \delta \tilde{p}$, and $\delta \tilde{v}_{T}$ as functions of the normalize 


\section{RYU AND VISHNIAC}

tually fragment. Although there have been numerical studies of the nonlinear evolution of perturbations of wind-driven bubbles (e.g., Rozyczka 1985), we argue that these are not reli- able because they fail to show the overstability in the early linear stage of evolution. In a subsequent paper, we will explore the nonlinear evolution for an infinitely thin shell.

\section{REFERENCES}

Vishniac, E. T. 1983, Ap. J., 274, 152.

Castor, J., McCray, R., and Weaver, R. 1975, Ap. J. (Letters), 200, L107. Rozyczka, M. 1985, Astr. Ap., 143, 59.

Ryu, D., and Vishniac, E. T. 1987, Ap. J., 313, 820

Sedov, L. I. 1959, Similarity and Dimensional Methods in Mechanics (New York: Academic).

Dongsu RyU and Ethan T. VishniaC: Department of Astronomy, University of Texas, Austin, TX 78712 